\title{
Thehazards of spacetravel
}

\author{
Before sending out astronauts on an interplanetary mission, weneed to investigatehow the conditionsin \\ spaceaffect human health. TheInternational SpaceStation is therefore of huge importanceto ensurethe \\ health of a spaceship crew travelling to other planets
}

\section{Richard B. Setlow}

$E^{\prime}$ xploration is an important survival strategy in evolution. The migration of expansive species depends on exploring their immediate or distant surroundings for new food sources or safe habitats; it can also come as a result of population pressures or environmental changes. The human species has added another reason for exploration, namely curiosity. This intellectual urge to explore the unknown led the great European explorers to the Americas, Australia and Antarctica between the fifteenth and seventeenth centuries. Inquisitiveness about nature is also the driving force behind humans exploring the polar caps, climbing mountain peaks and diving into the abysses of the oceans. Now, the ultimate frontier to explore in the twenty-first century is space. Astronomical observations and satellites have already yielded immense knowledge about our solar system and the universe beyond. But these technologies can provide only a limited picture of what is out there; eventually humans themselves will have to travel to other planets to investigate them in more intimate detail. Tremendous advances in rocket and spaceship technologies during the past 50 years, driven mainly by national security considerations, the need for better communication or a desire to observe environmental changes and human activity on the ground, have made it possible to send humans into near-Earth orbit and to the Moon. Conceivably, these advances will eventually make it possible to transport astronauts to other planets, and $\mathrm{M}$ ars in particular (Fig. 1).

But there are significant differences between exploring Earth and exploring space. First and foremost, space is an unforgiving environment that does not tolerate human errors or technical failure. For humans leaving Earth's orbit for extended periods, there are even more dangers. 0 ne is

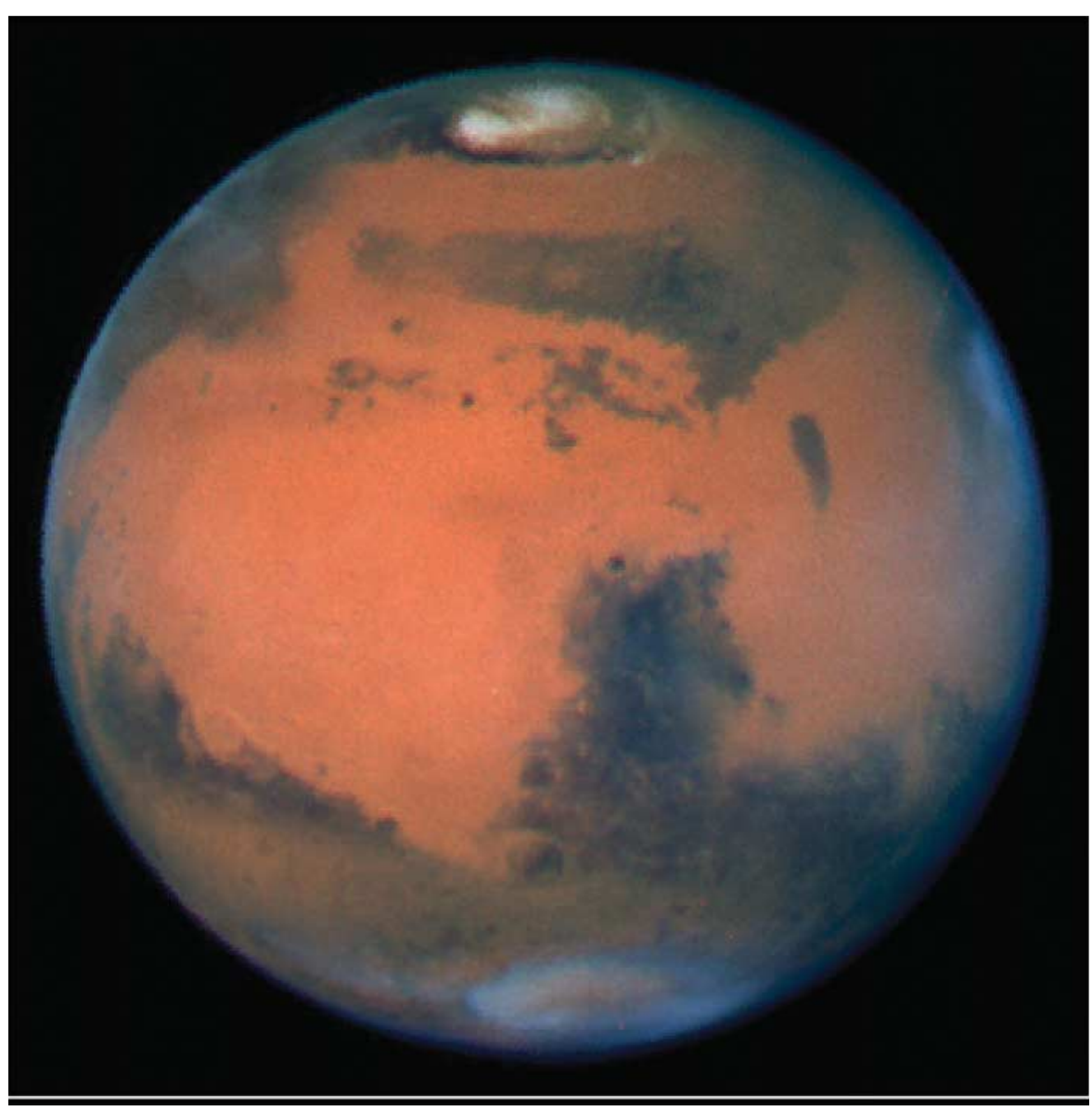

Fig. 1| Latespring on M ars. Source: NASA.

the near absence of gravity in space; the presence of high-energy, ionizing cosmic ray (HZE) nuclei is another. Because both zero gravity and cosmic rays would have severe health implications for astronauts on a Marsbound spaceship, we first need to investigate their effects on cells, tissues and our hormonal and immune systems. However, although we are able to produce HZE nuclei on Earth and study their effects on biological material, we cannot simulate extended periods of low gravity and their additive effects on cells and tissues. Thus, the International Space Station (ISS) will have an enormously important role in assessing the health dangers for humans in space and in the development of potential countermeasures.

T here is much information on the adaptation of astronauts to zero gravity $(0 \mathrm{~g})$ in space and on their return to $1 \mathrm{~g}$ on Earth. Nevertheless, our understanding of these effects is not complete; nor have countermeasures to mitigate them been identified. 


\section{As both zero gravity and cosmic rays would have severe health implications for astronauts on a Mars-bound spaceship, we first need to investigate their effects on cells, tissues and our hormonal and immunesystems}

Observations of astronauts travelling on the Space Shuttle and Russian cosmonauts' longterm visits to the Mir space station indicate that time spent in $0 \mathrm{~g}$ has serious effects on bone and muscle physiology and the cardiovascular system. For instance, the return from $0 \mathrm{~g}$ to $1 \mathrm{~g}$ leads to an inability to maintain an appropriate blood pressure when in an upright position-orthostatic intoleranceand insufficient blood flow to the brain. Astronauts returning from orbit therefore have to rest for several minutes, and the time needed to normalize their blood pressure increases with the time spent in $0 \mathrm{~g}$. This could mean that astronauts travelling to Mars- which would take at least one year in og-would need considerable time to readapt to gravity after landing there or after their return to Earth, unless we find a technological solution to the creation of artificial gravity on a spaceship. Moreover, there are other cardiovascular effects, such as cardiac arrhythmia and atrophy, that need to be studied in more detail before we can ensure the safety of astronauts on a M ars mission. O ther effects of extended time in low gravity are loss of bone mass and muscle deterioration. Without adequate countermeasures, these could impair the ability of astronauts to perform necessary functions on a spacecraft or on the surface of Mars.

T he second main danger for human travellers is the presence of the aforementioned $\mathrm{HZE}$ nuclei in cosmic rays, because of the ionizing effect that they exert on atoms or molecules. Although they do not reach the Earth's surface because they are either absorbed by the atmosphere or deflected by Earth's magnetic field, there are already some experimental data on the cancer-inducing properties of electrons, neutrons and protons in cosmic rays and other potential deleterious effects on biological material from numerous Earthbased experiments on laboratory animals. In addition, studies of the effects of the atomic bombs dropped on Japan in 1945 provided further data about the health dangers of radiation and high-energy nuclei.
However, cosmic rays are quite different from nuclear explosions because they include considerably higher numbers of HZE nuclei-leftovers from collapsing stars and supernova explosions that were thrown into space. Curtis \& Letaw (1989) estimated that on a three-year $M$ ars mission, about $30 \%$ of cells in the body will be traversed by HZE nuclei with $Z$ values- the number of protons-between 10 and 28 , and that virtually all cells will be traversed by nuclei with $Z$ values between 3 and 9 . These traversals result in numerous ionizing events, or hits, on cellular molecules. The biological effects of HZE nuclei on cancer induction, the central nervous system, the immune system and the eyes are not well known, nor have the interaction of radiation effects at $0 \mathrm{~g}$ been studied. Consequently we need to conduct many more experiments on Earth as well as on the ISS before the health and safety of astronauts travelling to Mars and beyond can be assured.

A nother, not yet seriously investigated, problem in addition to the direct damage of cells is the more general effect of space radiation on the immune system. Todd et al. (1999) estimated that the probability of the immune system's being affected is equal to or even greater than the probability of inducing mutations. The problem of estimating these indirect radiation effects is compounded by the fact that the dose rates of HZE produced in experiments on Earth are relatively high, whereas the dose rates in space- except for the intermittent but rare solar flares-are rather low. However, even such low doses could create significant complications through what is described as the 'bystander effect' in which cells-bystanders-that are not directly affected by radiation can be affected by neighbouring cells that have been hit and die or are mutated. The mechanisms for such effects involve cell-cell communication and the release of toxic products from damaged cells. Thus, the effects on tissue can be significantly larger than those estimated from individual cells, especially at low doses that affect only a fraction of cells in a tissue. The dose-response curve might not be a straight line at all but concave downwards, implying that the risks at low doses could be even larger than the risks estimated at the higher doses used in experiments on Earth (Brenner \& Elliston, 2001). Consequently, additional experiments are needed to estimate the effects of low doses of HZE particles on single cells and biological tissues or model organisms.

Simple experiments with yeast and bacteria on orbiting spacecraft have shown that $0 \mathrm{~g}$ does not significantly affect radiation responses. But these experiments have not been performed on higher organisms or biological tissues to investigate a potential synergism between radiation, $0 \mathrm{~g}$ and the stress of space travel on the immune system. These have to wait for completion of the ISS, but any further expansion of the space station is now on hold because of a shortage of funding, mainly because construction costs were higher than initially estimated (NRC, 2003). As a result, the installation of numerous facilities, such as habitats for animals, a radiation source and a $1 \mathrm{~g}$ centrifuge, is well behind schedule, and animal experiments, such as the effects of $0 \mathrm{~g}$ on radiation effects, cannot be done.

ronically, the health dangers of radiation in space only became an issue when the potential dangers of material brought back from space were discussed. In 1975 I joined the Space Science Board of the US National Research Council (NRC) that considered, among other issues, the problem of whether objects returned from the Moon or elsewhere from space could harbour deleterious organisms that would be hazardous to life on Earth. The appropriate solution at that time was to isolate these objects and extensively sterilize them with $\mathrm{X}$-rays or

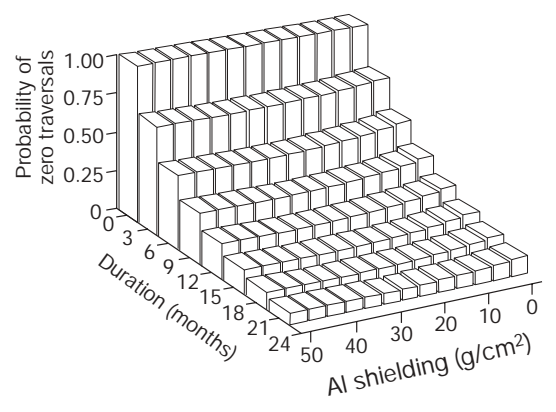

Fig. 2| Estimated probability that a nucleusin a blood-forming organ will not betraversed by the track core of a galactic cosmic ray, as a function of shielding and of duration in freespace. Figure adapted from Brenner \& Elliston (2001). 
ultraviolet radiation, or high temperatures. Because of my experience with various hazards of radiation exposure, I was subsequently asked to join the Committee on Space Biology and Medicine of the Space Science Board, and acted as chairman of its Task Group on the biological effects of space radiation. The committee produced a document entitled Radiation Hazards to Crews on Interplanetary Missions: Biological Issues and Research Strategies (NRC, 1996; Setlow, 1999) with various conclusions and recommendations. Two in particular are worth noting: 'The overall estimated uncertainty in the risks of radiation-induced biological effects ranges from a factor of 4- to 15fold greater to a factor of 4- to 15-fold smaller than our present estimates because of the uncertainties both in the way HZE particles and their spallation products penetrate shielding and the quantitative way in which these types of radiation affect biological functions', and 'U nless NASA obtains access to a reliable source of HZE particles ... for a significant fraction of each year, it will take over 10 years, perhaps over 20 years ... to reduce the present large uncertainties in particle transport behaviour and in the biological response functions'.

\section{Ironically, the health dangers of radiation in space only became an issue when the potential dangers of material brought back from space were discussed}

In response to the report, NASA and the Brookhaven National Laboratory (BN L) established a NASA-funded facility at BNL, the NASA Space Radiation Laboratory (NSRL), to produce HZE nuclei that mimic radiation in space. Commissioned in July this year, the NSRL will conduct ground-based experiments to determine the biological effects of HZE nuclei and test appropriate countermeasures to minimize the radiation levels in a space vehicle. One obvious way to reduce the number of $\mathrm{HZE}$ nuclei traversing a spacecraft would be to incorporate appropriate shielding. The usual types of shielding one thinks of would be heavy metals such as lead, or a lighter metal such as aluminium. However, although the flux of cosmic ray particles is readily

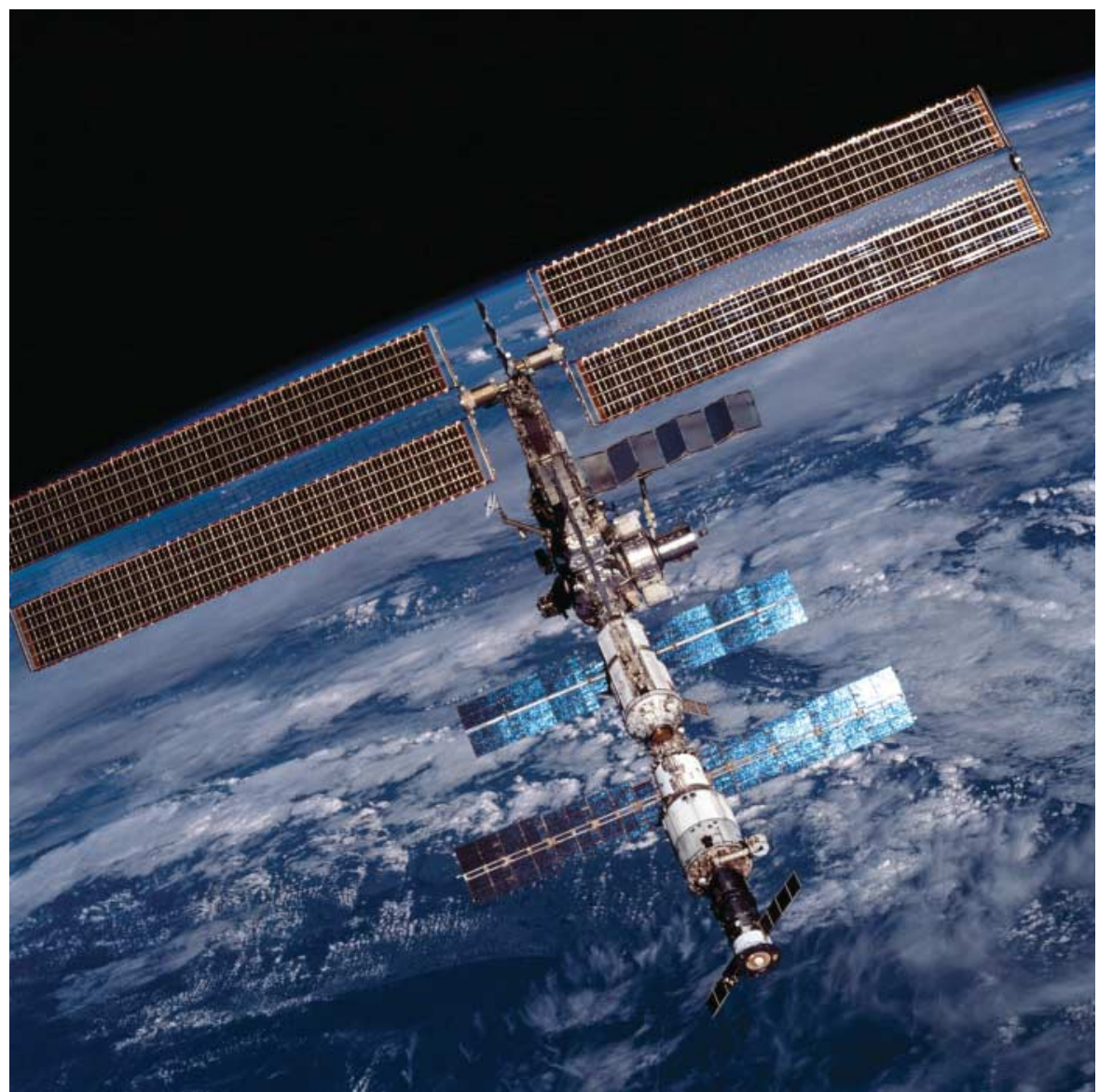

Fig. 3| The International Space Station as of 20 August 2001. Source: NASA.

attenuated by such shields, the particles split the nuclei in the shield, which produces energetic spallation productslower-mass nuclei-that also ionize and act as an additional source of radiation. Figure 2 shows the fraction of cells that escape traversal by HZE particles as a function of the mass of an aluminium shielding and the time being spent in space (Brenner \& Elliston, 2001). The longer the time spent in space, the more cells are hit- note that the mass of shielding has only a negligible effect. Lead would be an even less effective shielding material than aluminium; in general, metals are very poor countermeasures to protect against radiation. Lighter elements, such as water or plastics, might therefore be much better shields without adding additional mass to a spaceship. Future experiments to be performed at the NSRL will provide further evidence on different shielding materials and their effects on biological systems. nderstanding and evaluating the physiological effects of radiation and gravity require not only experiments on Earth but also extensive research on the ISS with an adequate number of animals and/or human subjects (Fig. 3). However, further expansion and work on the ISS has been stalled because of cuts in funding by NASA and, more recently, by the loss of the Columbia space shuttle in February this year. In addition, the ISS faces employment problems. O riginally, a crew of six or seven astronauts was planned for the ISS to maintain and run the station and to do scientific experiments. However, the shortage of funds means that there are not enough large space vehicles, such as space shuttles, available to transport crew, equipment and supplies and to serve as a rescue vehicle in case of a serious accident on the ISS. Hence, for safety reasons the crew size was reduced in 2002 to three, because only the Russian spacecraft, Soyuz, was available and that can carry only three crew members in an emergency. 


\section{science $\&$ society}

\section{Another problem in addition to the direct damage of cells is the more general effect of space radiation on theimmunesystem}

The loss of the Columbia shuttle in February 2003 has exacerbated this problem. As the crew size has been decreased from six to three, most of the astronauts' time will be spent on operation and maintenance of the station, which leaves little time for conducting scientific experiments. Without a significantly large infusion of funds to supply the equipment and to support a larger crew, the collection of basic information about the hazards of space travel will not be accomplished within the next 10-20 years. We also need a continuing, rotating crew of at least six astronauts to obtain epidemiologically significant data on the physiological and psychological effects of $0 \mathrm{~g}$ on astronauts and the efficacy of countermeasures. Unless these experiments can be done, it will not be possible to guarantee the safety and wellbeing of astronauts on a three-year trip to Mars and back.

So, how can we satisfy our curiosity about the Solar System and beyond, and continue to investigate the nearest planets in more detail? There are three possible solutions. The first, and most obvious, is to use unmanned spacecraft to investigate the planets' surface and to land, for example, on Mars or Europa-one of Jupiter's moons-and return samples to Earth. This might very well be done within the next
10 years. The second solution is to provide massively increased funding for the ISS. I cannot guess how much this would be, because, judging from past experience, there are large uncertainties in such estimates. And these funds would even eclipse the amount of money needed for a spacecraft that could transport a crew of six or seven astronauts on a three-year trip to $M$ ars and back. In the present global economic circumstances, this is certainly not feasible without significant physical and financial collaboration and cooperation among many countries. The third possible solution is to construct new lift-off capabilities and a much faster spacecraft to drastically reduce the time being spent in space and thus the radiation exposure (Fig. 2) and other stresses on astronauts. Science (2003) reported that Russia is working on plans for a nuclear-powered spacecraft to accomplish this goal. However, it is hard to envisage take-off and landing scenarios that would satisfy environmental concerns. Given the current situation, I therefore think that we will need to upgrade the ISS further and will have to stick with robot probes for at least the next 15 years before we can re-evaluate the rationale for sending humans to $\mathrm{M}$ ars.

\section{REFEREN CES}

Brenner, D.J. \& Elliston, C.D. (2001) The potential impact of bystander effects on radiation risks in a mars mission. Radiat. Res., 156, 612-617.

Curtis, S.B. \& Letaw, J.W. (1989) Galactic cosmic rays and cell-hit frequencies outside the magnetosphere. Adv. Space Res., $\mathbf{9}$ 293-298.

Science (2003) N ews Focus: O ne nuclear leap to Mars? Science, 301, 906-909.

N RC (1996) Radiation Hazards to Crews on Interplanetary M issions: Biological Issues and Research Strategies. N ational Academy Press, Washington, DC, USA.

N RC (2003) Factors Affecting the U tilization of the International Space Station for Research in the Biological and Physical Sciences. National Academy Press, Washington, DC, USA. <http://www.nap.edu>

Setlow, R.B. (1999) The U.S N ational Research Council's view of the radiation hazards in space. Mutat. Res., 430, 169-175.

Todd, P., Pecaut, M.J. \& Fleshner, M. (1999) Combined effects of space flight factors and radiation on humans. M utat. Res., $\mathbf{4 3 0}$ 211-219.

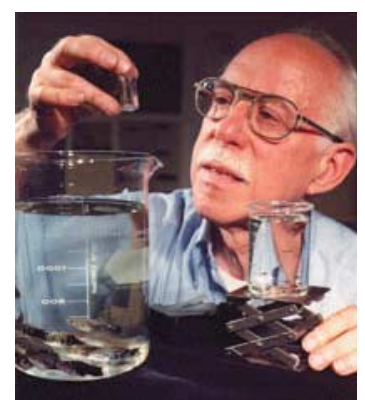

Richard B. Setlow is a Senior Biophysicist at the Brookhaven National Laboratory, U pton, New York, USA. The Laboratory is operated by Brookhaven ScienceAssociated, LLC. for the US D epartment of Energy. E-mail: setlow@bnl.gov

doi:10.1038/sj.embor.7400016 